\title{
CEST ANALYSIS: AUTOMATED CHANGE DETECTION FROM VERY-HIGH-RESOLUTION REMOTE SENSING IMAGES
}

\author{
Manfred Ehlers ${ }^{\mathrm{a}}$, Sascha Klonus ${ }^{\mathrm{a}}$, Thomas Jarmer ${ }^{\mathrm{a}}$, Natalia Sofina ${ }^{\mathrm{a}}$, Ulrich Michel ${ }^{\mathrm{b}}$, Peter Reinartz ${ }^{\mathrm{c}}$, Beril Sirmacek ${ }^{\mathrm{c}}$ \\ ${ }^{a}$ Institute for Geoinformatics and Remote Sensing, University of Osnabrueck, 49076 Osnabrueck, Germany \\ (mehlers, sklonus, tjarmer, nsofina)@igf.uni-osnabrueck.de \\ b University of Education, Department of Geography, Heidelberg, Germany (e-mail: michel@ph-heidelberg.de) \\ ${ }^{c}$ German Aerospace Center DLR, Remote Sensing Technology Institute, Wessling, Germany (e-mail: peter.reinartz, \\ beril.sirmacek@dlr.de)
}

KEY WORDS: Change Detection, Disaster, Texture, Visualization, Principal Component Analysis

\begin{abstract}
A fast detection, visualization and assessment of change in areas of crisis or catastrophes are important requirements for coordination and planning of help. Through the availability of new satellites and/or airborne sensors with very high spatial resolutions (e.g., WorldView, GeoEye) new remote sensing data are available for a better detection, delineation and visualization of change. For automated change detection, a large number of algorithms has been proposed and developed. From previous studies, however, it is evident that to-date no single algorithm has the potential for being a reliable change detector for all possible scenarios. This paper introduces the Combined Edge Segment Texture (CEST) analysis, a decision-tree based cooperative suite of algorithms for automated change detection that is especially designed for the generation of new satellites with very high spatial resolution. The method incorporates frequency based filtering, texture analysis, and image segmentation techniques. For the frequency analysis, different band pass filters can be applied to identify the relevant frequency information for change detection. After transforming the multitemporal images via a fast Fourier transform (FFT) and applying the most suitable band pass filter, different methods are available to extract changed structures: differencing and correlation in the frequency domain and correlation and edge detection in the spatial domain. Best results are obtained using edge extraction. For the texture analysis, different 'Haralick' parameters can be calculated (e.g., energy, correlation, contrast, inverse distance moment) with 'energy' so far providing the most accurate results. These algorithms are combined with a prior segmentation of the image data as well as with morphological operations for a final binary change result. A rule-based combination (CEST) of the change algorithms is applied to calculate the probability of change for a particular location. CEST was tested with high-resolution satellite images of the crisis areas of Darfur (Sudan). CEST results are compared with a number of standard algorithms for automated change detection such as image difference, image ratioe, principal component analysis, delta cue technique and post classification change detection. The new combined method shows superior results averaging between $45 \%$ and $15 \%$ improvement in accuracy.
\end{abstract}

\section{INTRODUCTION}

For change detection from remotely sensed images many methods have been proposed and developed. An overview and comparison of different change detection methods can be found in Singh (1989); Lu et al. (2003); or Coppin et al. (2004). In generally, change detection methods can be divided into three categories (Mas 1999): (i) Image enhancement-methods, (ii) multitemporal analysis, and (iii) post classification comparison. Other approaches combine several methods or consist of novel methodologies (an overview can be found in Lu et al. (2003)). Image enhancement methods are based on unclassified image data which combine the data mathematically to enhance the image quality. Examples of these are image difference, image ratio, or principal component (PC) and regression analysis. Multitemporal analysis methods are based on an isochronic analysis of multitemporal image data. This means that $n$ bands of an image taken on date $\mathrm{T} 1$ and $\mathrm{n}$ bands of an image of the same area taken on date $\mathrm{T} 2$ are merged to form a multitemporal image with $2 \mathrm{n}$ bands. This merged image is then used to extract the changed areas (Khorram et al. 1999). Post classification change analysis is based on a comparison of two independently generated classification results for at least two dates T1 und T2. In addition to simple change detection, this method also provides a change analysis; i.e., to determine the kind of change. It is, however, very sensitive to the achieved classification accuracy.
The large number of publications that deal with automated or semi-automated change detection prove that this field is an important research topic. Prakash \& Gupta (1998), for example, combine an image difference approach with vegetation indices. $\mathrm{Lu}$ et al. (2003) merge image difference with a principal component analysis. Dai \& Khorram (1999) use neural networks, whereas Foody (2001) and Nemmour \& Chibani (2006) involve fuzzy-set theory for change detection. Other approaches are based on object-based image analysis (Im et al. 2008). In summary, a wide range of different methods have been developed. These methods have a different grade of flexibility, robustness, practicability, and significance. Most authors, however, agree that there exist no single best algorithm for change detection. Therefore, new methods are still being developed and/or adapted especially for the detection of damaged buildings and infrastructure in conflict or crisis areas. This paper is no exception to this, as it described the development of, and the results for, a set of new change detection algorithms. They were tested with very high resolution (VHR) satellite images of the Dafur conflict area in Sudan. Multitemporal images of the affected regions were recorded by Quickbird-2 and are displayed on a web site that is hosted by Amnesty International clearly showing the destruction for a number of villages (http://www.eyesondarfur.org/villages.html). With the permission of the satellite company Digital Globe, we were able to use these preprocessed georeferenced Quickbird data that 
were acquired before and after an attack for our change analysis.

A fast detection and visualization of change in areas of crisis or catastrophes are important requirements for planning and coordination of help. Therefore, the objective of our research was to develop a reliable and accurate automated algorithm to detect changes on man-made objects. This algorithm should be used in catastrophic events or humanitarian crises to show the impact of this particular event..

\section{STANDARD CHANGE DETECTION METHODS}

For a comprehensive assessment of the quality of any new method it is essential to compare it to the performance of standard change detection approaches. For comparison, we selected those algorithms that are available in most remote sensing image processing systems. These methods are: (i) image difference; (ii) image ratio; (iii) PCA; (iv) delta cue; and (v) post classification analysis.

Image difference is an easy-to-understand and to-implement method. It is based on calculating the per-pixel gray value differences. If the resulting values are unchanged or do not exceed a pre-determined threshold no change has occurred. The degree of change is determined by the gray value differences. The image ratio method is very similar to image difference. For every pair of gray values at the same location at dates T1 and $\mathrm{T} 2$ the per-pixel ratio of the two values is calculated. Both methods vary through different spectral band combinations, the choice of thresholds, or different available spectral resolutions (Jensen 2005).

The principal component (PC) transform is a statistical method to calculate a new synthetic (uncorrelated) data space. PC analysis (PCA) can be used in different ways for change detection. In this study, we employ a selective bitemporal PCA (Tomowski et al. 2010). Two bitemporal spectral bands of the same location are analyzed in a two-dimensional feature space. As a result, all gray values are analyzed in relation to the two principal components. Usually, the unchanged pixels lie in the direction of the first PC whereas the changed pixel along the $2^{\text {nd }}$ PC axis.

Post classification analysis is based on a comparison of two independent classification results for at least two dates $\mathrm{T} 1$ und T2. This method allows the determination of the kind of change from one class to another.

The delta cue approach is a combination of different image processing techniques. These techniques are assembled into an integrated procedure. It consists of the following change detection algorithms: (i) tasseled cap soil brightness and greenness differences; (ii) magnitude difference; (iii) primary color difference; (iv) single band difference; and (v) band slope difference.

The following formula is used by all the presented change detection algorithms to compute the relative difference (R) of the images $\mathrm{T} 1$ and $\mathrm{T} 2$ :

$$
\mathrm{R}=\frac{\mathrm{T} 1-\mathrm{T} 2}{|\mathrm{~T} 1|}+\frac{\mathrm{T} 1-\mathrm{T} 2}{|\mathrm{~T} 2|}
$$

The features tasseled cap, primary color difference, band slope difference, and magnitude difference cannot be used in this study because the input images are panchromatic (single-band) images. This leaves just the single band difference algorithm and is therefore quite limited. In the next step, a threshold is determined to differentiate between real change and pseudo change. New geometric properties are then used to identify the changed buildings. These geometric properties include area, elongation, and compactness of connected pixels. These connected pixels build a blob of which major and minor axis can also be determined.

\section{COMBINED EDGE SEGMENT TEXTURE (CEST) ANALYSIS FOR CHANGE DETECTION}

Based on the fact that simple methods such as image differencing or image ratio failed to reliably detect changes of buildings in the study images, we had to develop a different procedure for automated change detection. This procedure is based on a number of different principles, namely frequency based filtering, segmentation, and texture analysis. Four of these methods are based on filtering in the frequency domain after a Fourier transform (FT), one on segmentation and the others on texture features. The frequency domain is used because it allows the direct identification of relevant features such as edges of buildings. If no features are directly visible (such as partial destruction with still standing outside walls), texture parameters are used for debris identification. A segmentation algorithm is used to extract size and shape of buildings. These methods can be combined in a decision tree for accuracy improvement. The combination of these processing steps is called Combined Edge Segment Texture (CEST) analysis.

\subsection{Fourier Transform Based Algorithms}

The FT is defined for a single band or panchromatic images (Cooley \& Tukey 1965). Based on a frequency analysis in the spectral domain, isotropic band pass filters can be designed that highlight selected frequencies and - as such - structures in the images. The design of band pass filters in the frequency domain is based on size and resolution of the images, and the estimated size of buildings and man-made structures where changes are to be detected. The filtered images are then transformed back into the spatial domain for further analysis. Higher frequencies visualize the position of building, the highest frequencies, however, contain mostly noise and are not useful for object identification and extraction. Lower frequencies contain mostly general image background which is not used for further analysis. After a number of tests, an optimum band pass filter is created which includes the most appropriate information for building extraction (Klonus et al. 2011b).

After transforming $\mathrm{T} 1$ and $\mathrm{T} 2$ via a fast FT (FFT) and the adaptive band pass filtering, four different methods can be applied to extract the changed structures: (i) subtraction in the frequency domain, (ii) correlation in the frequency domain, (iii) correlation in the spatial domain, and (iv) edge detection in the spatial domain. Of these methods, the best results are obtained using the edge detection algorithm (Klonus et al. 2011a). Consequently, we incorporated this method as a default function into the CEST analysis.

\subsection{Methods Based on Texture Parameters}

Frequency based filtering is particularly suited to detect changes in edge structures. If edges remain intact, however, textural features may be used for change analysis. For the calculation of texture parameters, we make use of the wellknown features defined by Haralick et al. (1973). The idea is that buildings can have higher texture values than areas without buildings, especially, if the surrounding neighborhood is very homogeneous and the buildings are very small or destroyed (with surrounding debris). The Haralick features are calculated using a window technique. Initial tests with a number of different features showed that 'energy' and 'inverse distance 
moment' (IDM, also known as 'homogeneity' ) produced the best results for man-made objects (Ehlers \& Tomowski 2008; Tomowski et al. 2010). Consequently, these features together with a bitemporal PCA were used for the CEST method.

\subsection{Change Detection Based on Segmentation}

Object or segment based image analysis has gained a lot of interest in the remote sensing community (see, for example, Otsu 1979 or Blaschke et al. 2008). Segmenting an image seems to be an excellent pre-analysis tool, especially for images of very high resolution. Consequently, we developed a segmentation procedure based on Euclidean distance to be used for change detection. For each pixel, the Euclidean distance to each neighboring pixel is calculated. If the distance is below a threshold, they belong to the same segment. After an independent segmentation of the images at dates $\mathrm{T} 1$ and $\mathrm{T} 2$, the segments of T1 are selected and used also for the T2 image. For each segment, the T1-T2 correlation coefficient is calculated. The result is assigned to each pixel in the segment. A new layer with the result of this segmentation is then created. Segments with a high correlation represent no changes. Segments with a low correlation represent changes.

This step is repeated for the opposite direction (i.e., T2-T1 correlation). The results are combined using different conditional statements. If, for example, the $\mathrm{T} 1$ image contains a number of buildings in a specific area which are not present in the T2 image, there exists a high probability that this area forms a large segment in T2 but is split into several small segments in T1. This would create incorrect change indications. As a final step, thresholds are used to extract the change segments.

\subsection{Combined Change Detection: The CEST Method}

Finally, all three methods are combined in a decision-tree approach (Fig. 1). The basis for the classification is the result of the change detection algorithm using edge detection based on frequency filtering. If the edge parameter indicates 'no change', the pixel in the image is classified as 'no change'. If the edge parameter indicates 'new building', the pixel is classified as new, if the texture feature 'energy' is an agreement. If energy indicates 'change' and one of the features 'homogeneity' or 'segmentation' indicate 'change', the result is 'new'. Otherwise, it is classified as unchanged. If the edge parameter shows 'change', it is classified as 'change' if the texture feature 'energy' coincides. If energy indicates 'no change', the pixel will be classified as 'no change'. If energy indicates 'new' but the segment and homogeneity parameters show 'change', the pixel is assigned to 'change'. Otherwise it is classified as unchanged. The CEST procedure was tested against the standard change detection methods described above.

\subsection{Automatically Created Damage Maps}

The produced change images are to a large degree abstract and hard to interpret. This holds particularly true for people not accustomed to remote sensing such as members of official organizations or rescue forces. For the purpose of planning after a crisis or a catastrophe, the interpretation of change images should be as easy as possible. An algorithm was developed to automatically produce a map which can be easily interpreted. The first step is to generalize the change image. Inside a $20 \times 20$ pixels window, the amount of change is determined using the information in the change image. The change percentage of this area is calculated and then ranked into a number of distinctive general classes. If less than $15 \%$ of the area has changed, all pixels are classified as unchanged. Change above $80 \%$ marks extensive change and change between $15 \%$ and $80 \%$ marks low to moderate change. Areas of new buildings with a surface cover of at least $15 \%$ are indicated as 'new areas'.

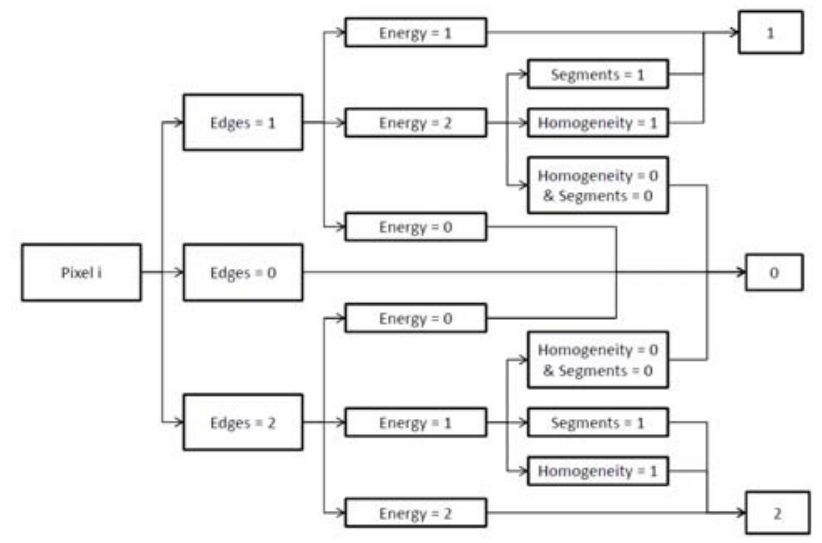

Fig. 1. CEST decision tree (description see in the text)

\section{STUDY AREA}

CEST detection and change map generation methods are now applied to the selected study sites in Darfur, Sudan. They represent areas which experienced dramatic changes during the Darfur conflict. It is estimated that more than 300.000 people have already died in this conflict and more than 2 million people have been displaced (http://www.eyesondarfur.org).

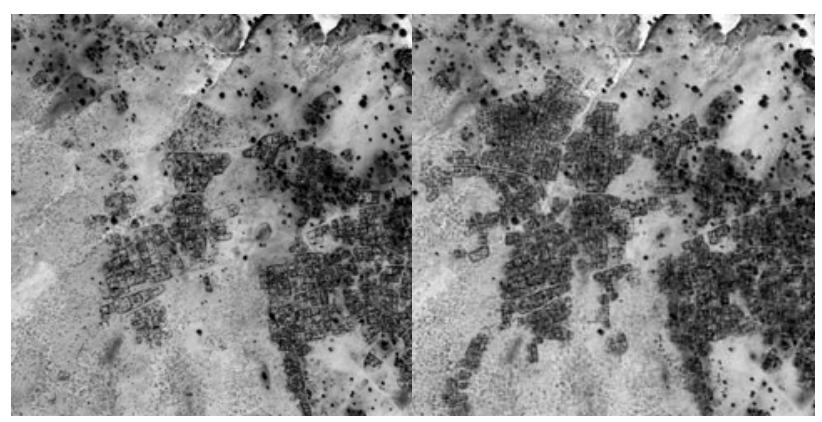

Fig. 2 Panchromatic Quickbird-2 images recorded on March 2, 2006 (left - before the attack) and on February 28, 2008 (rightafter the attack) of the town Abu Suruj (2048 x 2048 pixels). Images courtesy of Digital Globe.

The test area is located in South Darfur and shows part of the town Abu Suruj in West Darfur (Fig. 2). Because of destroyed and new settlement areas, this study site is very complex. It contains changes due to destruction and - at the same time changes due to construction. A change detection procedure should be capable of depicting both types of change. For a more detailed look at the conditions, Fig. 3 shows subsets of Fig. 2. A manually digitized change image (black = no change, gray = destroyed, white = new) which will be used as 'ground truth' is displayed in Fig. 4.

A visual comparison and overlay of the existing man-made structures shows a high correspondence for both images, so that a new co-registration was not necessary and the problem of possible pseudo change was negligible. They were preprocessed using a histogram matching procedure. An atmospheric correction is not applied, due to missing ground truth data, sparse vegetation and only one image band. 


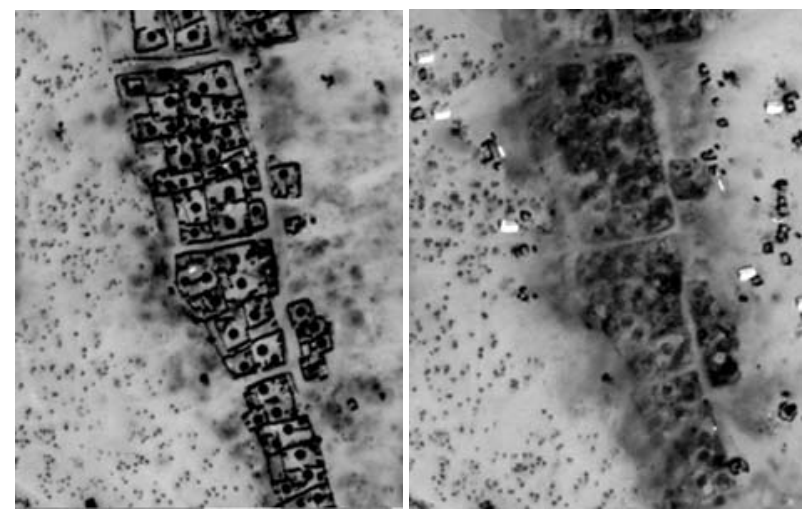

Fig. 3 Subsets of the Quickbird images of 2006 and 2008, respectively (280 x 350 pixels)

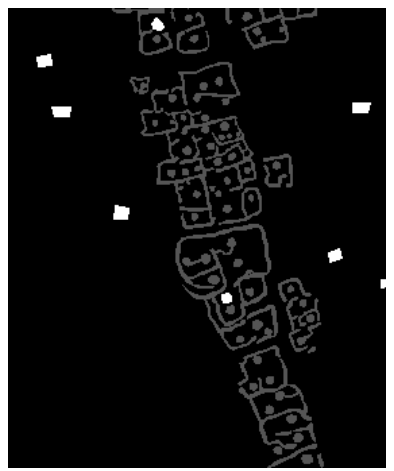

Fig.4 Manually digitized change image

\section{RESULTS AND ACCURACY ASSESSMENT}

In the following section, we present the results of the standard algorithms, the new CEST method, and the achieved accuracies. For the accuracy assessment three classes were selected:

Class $0=$ unchanged buildings/background (black)

Class 1 = changed or destroyed buildings (gray)

Class 2 = new buildings (white)

The reference is the manual digitization of Fig. 4. Accuracy assessment for classes 1 and 2 is based on 404 randomly chosen digitized objects. Only for class 0 all 404 objects were used. If the majority of the pixels inside an object are assigned the correct class, the whole object is considered as correctly detected. Producers' accuracy, users' accuracy and the kappa coefficients are calculated for all scenarios.

\subsection{Image Difference and Image Ratio}

For image difference, it is possible to detect the three different classes (positive change, negative change and no change). It can be seen, however, that large areas of pseudo change are detected (Fig. 5 left). Due to brightness changes of the sediment, change is especially detected in the north of the image. Most of the new buildings which appear in the T2 image are detected. Buildings which are unchanged are often identified as destroyed or changed buildings. For image ratio, it is difficult to find a threshold between new and changed/destroyed buildings. Therefore most of the buildings are detected as new buildings (Fig. 5 right). As with image difference, buildings which are unchanged are often detected as destroyed or changed. This leads to the extremely low producers accuracy of $8.2 \%$ for class 1 (changed or destroyed buildings). The amount of detected pseudo change is relatively low in comparison to image difference.
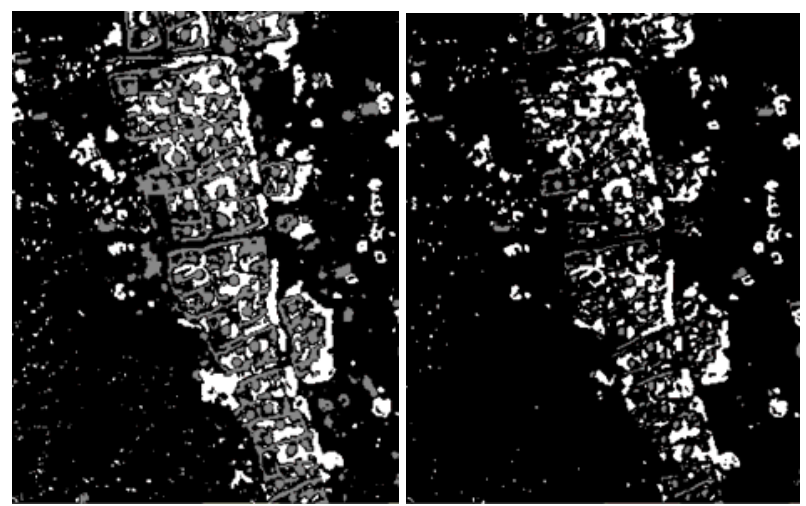

Fig. 5 Change detection by image difference (left) and image ratio (right)

\subsection{PCA}

The image processed with the PCA change detection procedure shows a lot of pseudo change, especially in the south and west of the image. Similar to the image ratio result, most of the buildings are detected as new buildings (Fig. 6 left). Also, nearly $45 \%$ of the unchanged buildings are classified as changed/destroyed. $30 \%$ of the destroyed or changed buildings, on the other hand, are classified as unchanged.

\subsection{Delta Cue}

The delta cue method produces a change image with relatively high producer accuracies for class 0 and 1 (Fig. 6 right). More than $60 \%$ of the unchanged buildings, however, were detected as changed/destroyed. Additionally, a large amount of pseudo change appears in the image, especially in the northeast.

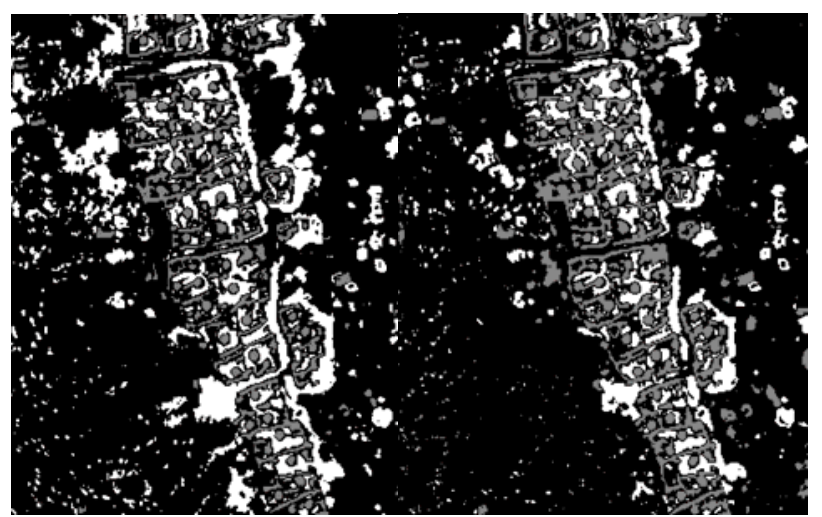

Fig. 6 Change detection by PCA (left) and delta cue (right)

\subsection{Post Classification}

For the post classification analysis we used the isodata algorithm, because no appropriate training areas were available. This method produces the lowest accuracies. Again, pseudo change poses a big problem (Fig. 7 left). 


\subsection{CEST}

The result of the four methods combined in the CEST approach is shown in Fig. 7 (right). In comparison to the other methods, this image contains far less noise. Also, misclassification of vegetation as changed buildings is significantly less. In addition, the walls of the buildings are more accurate than for the other results. In total, the combination of all three methods generates the most reliable and accurate results for change detection which is also demonstrated by the accuracy assessment. The $\kappa$-coefficients are presented in table 1 .

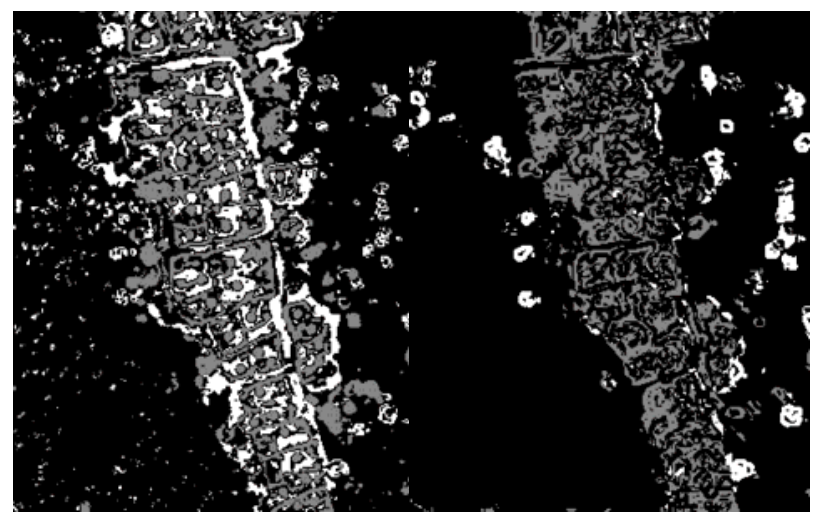

Fig. 7 Change detection by post classification (left) and CEST (right)

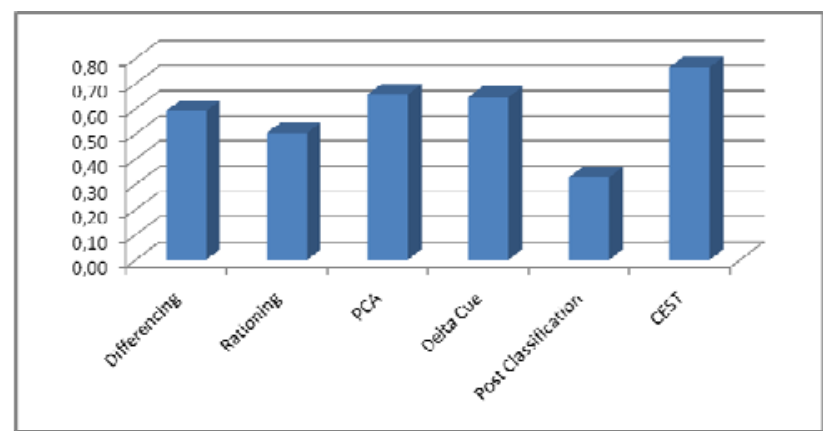

Tab. 1 к-coefficients for the change detection methods

\subsection{Damage Map}

For the damage map, the original image of $\mathrm{T} 2$ is used as background for the automatically created change maps. Unchanged areas are transparent, low to moderate changes are shown as yellow overlay, and areas of strong changes as red overlay. New building areas are shown in green. If this technique is applied to areas with catastrophic events, this change map makes it possible to quickly identify the most affected areas or the areas for which high casualties are likely. For the Abu Suruj area, it could be easily depicted that the town has increased, but also that large parts of the city have changed. Buildings were destroyed and new buildings were built on these sites or next to the destroyed buildings (Fig. 8).

\section{CONCLUSIONS}

In this paper, a new automated change detection method (CEST) is presented. CEST combines adaptive filtering in the frequency domain with edge detection in the spatial domain, calculation of the texture features 'homogeneity' and 'energy' with a PCA change detection approach and segment based correlation. This combined method is compared to five standard change detection algorithms (image difference, image ratio, PCA, delta cue, and post classification analysis). Results are visually and quantitatively analyzed. The accuracy assessment shows that the CEST method is far superior to the standard techniques for change detection. The combined method yields an overall accuracy of $80 \%$ and more than $90 \%$ of the unchanged buildings could be correctly identified. The method is also transferable to other scenes of Darfur.

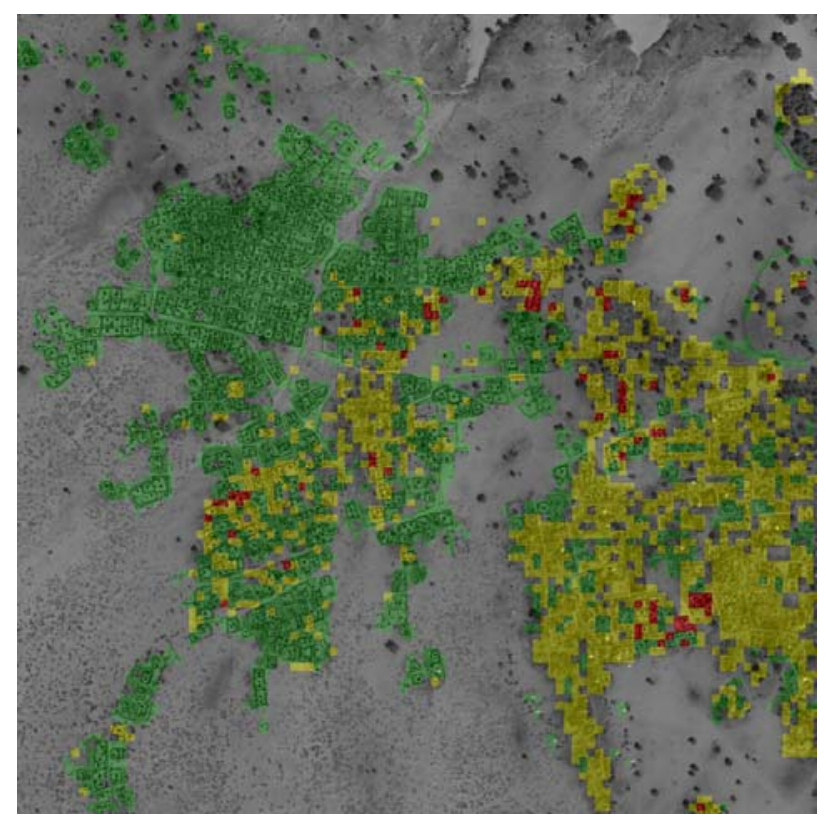

Fig. 8 Change map of Abu Suruj: new buildings (green), low to moderate change (yellow), extensive change (red).

\section{REFERENCES}

Blaschke, T., S. Lang \& G. Hay (Eds.), 2008. Object-Based Image Analysis - Spatial Concepts for Knowledge-Driven Remote Sensing Applications, Springer Lecture Notes in Geoinformation and Cartography, Heidelberg.

Cooley, J.W. \& J.W. Tukey, 1965. Raster algorithm for machine calculation of complex Fourier series, Mathematics of Computation, vol. 19, 297-301.

Coppin, P., I. Jonckheere, K. Nackaerts, B. Muys, \& E. Lambin, 2004. Digital change detection methods in ecosystem monitoring a review. International journal of remote sensing, 25(9), pp. 1565-1596.

Dai, X. \& S. Khorram, 1999. Remotely sensed change detection based on artificial neural networks, Photogrammetric Engineering and Remote Sensing, vol. 65, pp. 1187-1194.

Ehlers, M \& D. Tomowski, 2008. On segment based image fusion, in: Blaschke, T., S. Lang and G. Hayes (Eds.), ObjectBased Image Analysis - Spatial Concepts for KnowledgeDriven Remote Sensing Applications, Springer Lecture Notes in Geoinformation and Cartography, Heidelberg, pp. 735-754.

Foody, G.M., 2001. Monitoring the magnitude of land-cover change around the southern limits of the Sahara, Photogrammetric Engineering and Remote Sensing, vol. 67, pp. 841-84.

Haralick, R.M., K. Shanmugam \& I. Dinstein, 1973. Textural features for image classification, IEEE Trans. Syst., Man, Cybern., vol. 3, pp. 610-621. 
Im, J., J.R. Jensen \& J.A. Tullis, 2008. Object-based change detection using correlation image analysis and image segmentation, International Journal of Remote Sensing, vol. 29, pp. 399-423.

Jensen, J.R., 2005. Introductory digital image processing: A remote sensing perspective. Prentice-Hall, Englewood Cliffs, NJ.

Khorram, S., G.S. Biging, N.R. Chrisman, D.R. Colby, R.G. Congalton \& J.E. Dobson, 1999. Accuracy assessment of remote sensing derived change detection, ASPRS, Bethesda, MD.

Klonus, S., D. Tomowski, M. Ehlers, U. Michel \& P. Reinartz, 2011a, Change Detection mittels Fourieranalysen, segmentsowie texturbasierter Methoden, gi-reports@igf, vol. 3.

Klonus, S., M. Ehlers, D. Tomowski, U. Michel \& P. Reinartz, 2011b. Detektion von zerstörten Gebäuden in Krisengebieten aus panchromatischen Fernerkundungsdaten, Photogrammetrie - Fernerkundung - Geoinformation (PFG), pp. 219-231.

Lu, D., P. Mausel, E. Brondízio \& E. Moran, 2003. Change detection techniques, International journal of remote sensing, vol. 25, pp. 2365-2407.

Mas, F.J., 1999. Monitoring land-cover changes: a comparison of change detection techniques, International Journal of Remote Sensing, vol. 20, pp. 139-152.

Nemmour, H. \& Y. Chibani, 2006. Fuzzy neural network architecture for change detection in remotely sensed imagery, International Journal of Remote Sensing, vol. 27, pp. 705-717.
Otsu, N., 1979. A threshold selection method from grey level histograms, IEEE Transactions on Systems, Man, and Cybernetics, vol. 9, pp. 62-66.

Prakash, A. \& R.P. Gupta, 1998. Land-use mapping and change detection in a coal mining area - a case study in the Jharia coalfield, International Journal of Remote Sensing, vol. 19, pp. 391-410.

Singh, A., 1989. Digital change detection techniques using remote-sensed data, International Journal of Remote Sensing, vol. 10, pp. 989-1003.

Tomowski, D., S. Klonus, M. Ehlers, U. Michel \& P. Reinartz, 2010. Change visualization through a texture-based analysis approach for disaster applications, in: W. Wagner \& B. Szekeyk (Eds.), ISPRS Technical Commission VII Symposium - 100 Years ISPRS Advancing Remote Sensing Science, IAPRS Vol. XXXVIII, Part 7A, Vienna, pp. 263-269

Web sites:

http://www.eyesondarfur.org (last access: April 2012)

\section{ACKNOWLEDGEMENTS}

The presented work was done in the research project "Automated change detection methods for disaster applications" at the Institute for Geoinformatics and Remote Sensing (IGF) of the University of Osnabrueck. 